Research Article

Human and Medical Genetics

\title{
Epistasis of polymorphisms related to the articular cartilage extracellular matrix in knee osteoarthritis: Analysis-based multifactor dimensionality reduction
}

\author{
Javier Fernández-Torres ${ }^{1}$, Gabriela Angélica Martínez-Nava ${ }^{1}$, Yessica Zamudio-Cuevas ${ }^{1}$, Carlos Lozada $^{2}$, \\ Daniela Garrido-Rodríguez ${ }^{3}$ and Karina Martínez-Flores ${ }^{1}$ iD \\ ${ }^{1}$ Synovial Fluid Laboratory, Instituto Nacional de Rehabilitación "Luis Guillermo Ibarra Ibarra", Mexico \\ City, Mexico. \\ ${ }^{2}$ Rheumatology Service, Instituto Nacional de Rehabilitación "Luis Guillermo Ibarra Ibarra", Mexico City, \\ Mexico. \\ ${ }^{3}$ Center for Research in Infectious Diseases, National Institute of Respiratory Diseases, Mexico City, \\ Mexico.
}

\begin{abstract}
Osteoarthritis $(\mathrm{OA})$ is a complex disease with a multifactorial etiology. The genetic component is one of the main associated factors, resulting from interactions between genes and environmental factors. The aim of this study was to identify gene-gene interactions (epistasis) of the articular cartilage extracellular matrix (ECM) in knee OA. Ninety-two knee OA patients and 147 healthy individuals were included. Participants were genotyped in order to evaluate nine variants of eight genes associated with ECM metabolism using the OpenArray technology. Epistasis was analyzed using the multifactor dimensionality reduction (MDR) method. The MDR analysis showed significant gene-gene interactions between MMP3 (rs679620) and COL3A1 (rs1800255), and between COL3A1 (rs1800255) and VEGFA (rs699947) polymorphisms, with information gain values of $3.21 \%$ and $2.34 \%$, respectively. Furthermore, in our study we found interactions in high-risk genotypes of the HIF1AN, MMP3 and COL3A1 genes; the most representative were $[A A+C C+G A],[A A+C T+G A]$ and $[A A+C T+G G]$, respectively; and low-risk genotypes $[A A+C C+G G]$, $[G G+T T+G A]$ and $[A A+T T+G A]$, respectively. Knowing the interactions of these polymorphisms involved in articular cartilage ECM metabolism could provide a new tool to identify individuals at high risk of developing knee OA.
\end{abstract}

Keywords: Epistasis, extracellular matrix, knee osteoarthritis, multifactor dimensionality reduction, polymorphisms.

Received: November 29, 2018; Accepted: June 26, 2019.

\section{Introduction}

To date, knee osteoarthritis (OA) prevails as the main cause of physical disability in senior adults. It is well known that age, gender, overweight, and genetic predisposition are key factors for its development (Arden and Nevitt, 2006; Peláez-Ballestas et al., 2011; Hernández-Cáceres et al., 2015). Articular cartilage, synovial membrane, and subchondral bone are tissues affected by OA; however, other tissues, such as meniscus and ligaments are compromised too. Progressive degeneration of articular cartilage, the subsequent decrease in joint space and osteophyte formation, as well as proteoglycans and collagen loss, extracellular matrix (ECM) mineralization, and hypertrophic differentiation of chondrocytes represent the hallmark of OA (De Filippis et al., 2004; Bertrand et al., 2010; Kraus et al., 2015).

Send correspondence to Karina Martínez-Flores. Synovial Fluid Laboratory, Instituto Nacional de Rehabilitación "Luis Guillermo Ibarra Ibarra”. Calzada Mexico-Xochimilco 289, 14389, Mexico City, Mexico. E-mail: karinabiologist@ hotmail.com.
Healthy articular cartilage is a hyaline, viscoelastic, avascular, aneural, and alymphatic tissue. It is composed of an ECM rich in collagens (types II, IX and XI), proteoglycans (aggrecan), and water. The cellular part consists of chondrocytes only (Sophia et al., 2009). As a result of being an avascular tissue, oxygen and nutrients are lower than in other tissues, creating a hypoxic environment $(0.5-10 \%$ of oxygen, or $4-70 \mathrm{~mm} \mathrm{Hg}$, respectively, which also depends of the zone in tissue, either deep or superficial) (Pfander et al., 2006; Oswald et al., 2008; Ströbel et al., 2010). Despite the extreme conditions of the cartilage, hypoxia is necessary in metabolic processes, such as chondrogenesis ECM synthesis and degradation. It also modulates the subchondral bone angiogenesis through the vascular endothelial growth factor A (VEGFA). These processes, and the proper maintenance of general cartilage homeostasis, are coordinated by hypoxia-inducible factor-1 $\alpha$ (HIF-1 $\alpha$ ) (Pfander et al., 2006). If the ECM synthesis process is decreased, cartilage will become thinner and weaker, whereas it will turn hyper- 
trophic and disorganized with a ECM synthesis increase, as a way to compensate for the null ECM generated favoring osteophyte formation (Martel-Pelletier et al., 2008). In advanced life stages, or during the development of joint diseases, such as OA, the delicate balance between ECM synthesis and degradation is heavily affected by factors responsible for articular cartilage degradation, among which stand out the proinflammatory cytokines (IL-1 $\beta$, IL-6, IL-8, TNF- $\alpha$ ), chemokines, metalloproteinases (MMP-1, 3, 9 and 13), and aggrecanases (ADAMTS 4, 5 and 9) (Mariani et al., 2014). In addition to these molecules, EGFR ligands are able to stimulate the expression of genes related to cartilage catabolism and, on the other hand, inhibit the catabolic activity of chondrocytes (Long et al., 2015). Although VEGFA plays a beneficial role for the articular cartilage, during OA it is actively involved in remodeling the ECM and forming osteophytes (Hamilton et al., 2016). On the other hand, the concentration of nitric oxide $(\mathrm{ON})$ generated by nitric oxide synthase (NOS) regulates MMP synthesis. At low concentrations it inhibits production, but at high concentrations, it activates them, favoring ECM degradation. It also promotes free radical production and induces chondrocyte apoptosis (Abramson, 2008a,b).

From a genetic standpoint, several studies suggest associations between single-nucleotide polymorphisms (SNP) and knee OA (Fernández-Moreno et al., 2008; van Meurs and Uitterlinden, 2012; Fernández-Torres et al., 2017; Ozcan et al., 2017). Nevertheless, most of them were assessed individually, in contrast to joint assessments through genegene interactions (epistasis), which could provide more information regarding their role. Recently, Wang et al. (2016) published a meta-analysis associating different SNPs with knee, hip, and hand OA risk, and suggesting that genetic predisposition is a key factor for OA development. Another relevant aspect to be considered is the fact that SNP distribution in this kind of studies is greatly affected by ethnicity and geographic location.

Several studies related to the analysis of gene-gene interactions in complex diseases have only been conducted using logistic regression models, linkage disequilibrium, and Hardy-Weinberg equilibrium tests, all of which have limitations (Ritchie et al., 2003; Mechanic et al., 2008). Therefore, identification and characterization of gene-gene and geneenvironment interactions have been limited primarily due to a lack of powerful statistical methods, and particularly because of small sample sizes, which has been a challenge for geneticists. In this sense, the multifactor dimensionality reduction (MDR) method does not require a model as such, given that no genetic models are assumed, neither is it parametric, as no parameters are estimated.

Dimensionality is defined as the interactions between genetic or environmental factors, which increase exponentially as these factors increase. MDR reduces the dimensionality of multilocus data to improve the ability to detect genetic combinations that confer disease risk. MDR pools genotypes into 'high-risk' and 'low-risk' or 'response' and 'non-response' groups in order to reduce multidimensional data into only one dimension (dichotomous variable). Because it is a non-parametric method, no hypothesis concerning the value of any statistical parameter is made. Additionally, MDR was designed to detect gene-gene or gene-environment interactions in datasets with categorical independent variables, such as SNP and other sequence variations (insertions, deletions, etc.), as well as environmental data that can be represented as categorical variables. The endpoint, or dependent variable, must be dichotomous, such as case/control for studies of human disease. Pharmacogenomics data can also be analyzed with MDR, in terms of 'response/non-response' or 'toxicity/no toxicity'. MDR is appropriate for any data type with two distinct clinical endpoints (Motsinger and Ritchie, 2006; Moore and Andrews, 2015).

For studies with more than two factors, the steps of the MDR method are repeated for each possible model size (two-factor, three-factor, etc.), if computationally feasible. The result is a set of models, one for each model size considered. From this set, the model with the combination of loci and/or discrete environmental factors that maximize the cross validation consistency and minimize the prediction error is selected. Cross-validation consistency is a measure of the number of times an MDR model is identified in each possible 90 per cent of the subjects. When cross-validation consistency is maximal for one model and prediction error is minimal for another model, statistical parsimony is used to choose the best model. On the other hand, a balanced accuracy function is used when the ratio of cases to controls is different than one (Motsinger and Ritchie, 2006; Moore and Andrews, 2015).

The MDR method is based on an algorithm that generates the characteristics or attributes created by a new variable or attribute. It has also developed an entropy-based method for a better statistical interpretation of the results (Hahn et al., 2003; Cho et al., 2004; Gui et al., 2011; Su et al., 2015; Kohli et al., 2016). Previously, Su et al. (2015) performed an epistasis study between TGF- $\beta$ and Smad3 polymorphisms and OA patients, revealing that it can affect the development of OA. Recently, our work group published a study of epistasis between polymorphisms related to oxidative stress and OA patients, and the results have shown a strong interaction between ADIPOQ (rs1501299) and PON1 (rs662) polymorphisms (Fernández-Torres et al., 2019), suggesting an important role in the OA pathogenesis; both studies were carried out using the MDR method. The generalized MDR (GMDR) method is an extension from MDR. It allows an adjustment for discrete and quantitative covariables and can be applied to both dichotomous and continuous phenotypes in several study designs based on population. Additionally, a command sequence includes $p$-value assessment (Lou et al., 2007; Chen et al., 2011).

Interactions between multiple polymorphisms of different genes could be the foundation of the genetic origin of OA. Therefore, this study is focused on evaluating whether interactions between several genetic variants of articular car- 
tilage ECM are associated with knee OA in a Mexican population.

\section{Subjects and Methods}

\section{Subjects}

Two hundred thirty-nine unrelated individuals of Mexican ancestry and geographically matched were included in this case-control study. Ninety-two of them were knee OA patients of both genders, recruited from the Rheumatology Service of the Instituto Nacional de Rehabilitación "Luis Guillermo Ibarra Ibarra" (INR-LGII). Knee OA was diagnosed under the guidelines of the American College of Rheumatology (Altman et al., 1986); the clinical exam and the X-ray evaluation were performed by a rheumatologist and a radiologist, respectively. OA severity was evaluated using the Kellgren-Lawrence radiological scale (K-L) (Kellgren and Lawrence, 1957). Patients with $\mathrm{K}-\mathrm{L} \geq 2$ were included, and those with other etiologies causing knee diseases, such as inflammatory arthritis (rheumatoid arthritis or any other autoimmune disease), post-traumatic arthritis, post-septic arthritis, poliomyelitis, or skeletal dysplasia, were excluded. One hundred forty-seven healthy employees of the INR-LGII of both genders, with no signs or symptoms of knee OA, OA family history, and no other type of arthritis or joint painful condition were recruited as controls from the departments of Human Communications and Human Resources, as well as the cleaning staff. They were invited to participate in the study through the proper authority. Additionally, all evaluated controls showed a K-L grade equal to or lower than one. All participants were at least 40 years old and signed an informed consent. Data on age, gender, body mass index (BMI), and birth place was collected through an interview. This study was conducted under the criteria set forth in the Declaration of Helsinki and was approved by the INR-LGII Ethics and Research Committee (CONBIOETICA-09CEI-031-20171207).

\section{SNPs selection and genotyping}

For this study, nine candidate SNPs from eight genes involved in articular cartilage ECM were selected. The search strategy was as follows: a) information of each SNP was obtained from the public databases Hap Map (http://www.hapmap.ncbi.nlm.nih.gov/) and the National Center for Biotechnology Information dbSNP database (http://www.ncbi.nlm.nih.gov/snp); b) the previously selected SNPs evaluated in OA or other pathologies (Rodriguez-Lopez et al., 2006; Jacobs et al., 2008; Chen and Mattey, 2012; Zhan et al., 2013; Gibbon et al., 2017; Guo et al., 2017; Kaynak et al., 2017; Du et al., 2019); and c) the selected polymorphisms should not be in linkage disequilibrium between them. Finally, polymorphisms with minor allele frequency (MAF) in the Mexican population $<10 \%$ were excluded (Table 1). Selected SNPs that were genotyped in cases and controls were rs699947, rs3025039 (VEGFA), rs11292 (HIFlAN), rs1800255 (COL3A1), rs4444903 (EGF), rs679620 (MMP3), rs2252070 (MMP13), rs2297518 (NOS2), and rs2070744 (NOS3).

Given that the Mexican population structure is composed of a mix of Amerindian, European, and African ancestors, a set of nine ancestry-informative markers (AIMs) was used to evaluate whether any resulting association could be a confounder due to the population stratification during the data adjustment step (Bonilla et al., 2004; Choundry et al., 2006) (Table S1).

DNA was isolated from peripheral blood in EDTA tubes, using a commercial kit (QIAmp 96 DNA Blood Kit, Qiagen, Hilden, Germany). The samples were genotyped through the OpenArray technology on a QuantStudio 12K flex equipment (Thermo Fisher Scientific). Genomic DNA from the samples was adjusted to $50 \mathrm{ng} / \mu \mathrm{L} ; 2.5 \mu \mathrm{L}$ of DNA were mixed with $2.5 \mu \mathrm{L}$ of TaqMan OpenArray Genotyping Master Mix (Thermo Fisher Scientific) in 384-well trays. The mixtures were loaded onto OpenArray genotyping trays

Table 1 - Single-nucleotide polymorphisms (SNPs) studied.

\begin{tabular}{|c|c|c|c|c|c|c|c|c|}
\hline Closest gene & $\mathrm{db}$ SNP rs ID & Chromosome: position & Location & $\begin{array}{l}\text { MAF in Mexi- } \\
\text { can population }\end{array}$ & Disease & $\mathrm{OR}^{*}$ & $n$-size & Reference \\
\hline$V E G F A$ & rs699947 & Chr.6:43736389 & 5'UTR & $0.42(\mathrm{~A})$ & RA & - & 413 & Chen and Mattey, 2012 \\
\hline$V E G F A$ & rs3025039 & Chr.6:43752536 & 3'UTR & $0.30(\mathrm{~T})$ & $\mathrm{PC}$ & 0.91 & 1621 & Jacobs et al., 2008 \\
\hline$H I F 1 A N$ & rs11292 & Chr.10:102313607 & 3'UTR & $0.13(\mathrm{G})$ & $\mathrm{BC}$ & 1.12 & 1056 & Du et al., 2019 \\
\hline COL3A1 & rs 1800255 & Chr. 2:189864080 & Exon & $0.23(\mathrm{~A})$ & $\mathrm{ACL}$ & 0.78 & 321 & $\begin{array}{l}\text { Stepien-Soldowska et } \\
\text { al., } 2015\end{array}$ \\
\hline$E G F$ & rs4444903 & Chr.4:110834110 & 5'UTR & $0.38(\mathrm{~A})$ & $\mathrm{PC}$ & 0.98 & 1636 & Jacobs et al., 2008 \\
\hline$M M P 3$ & rs679620 & Chr.11:102713620 & Missense variant & $0.31(\mathrm{~T})$ & $\mathrm{OA}$ & 1.45 & 297 & Guo et al., 2017 \\
\hline$M M P 13$ & rs 2252070 & Chr.11:102826539 & 5'UTR & $0.29(\mathrm{C})$ & RA & 1.13 & 1202 & $\begin{array}{l}\text { Rodriguez-Lopez et } \\
\text { al., } 2006\end{array}$ \\
\hline NOS 2 & rs 2297518 & Chr.17:26096597 & Missense variant & $0.13(\mathrm{~A})$ & $\mathrm{PC}$ & 1.03 & 1637 & Jacobs et al., 2008 \\
\hline NOS3 & rs2070744 & Chr.7:150690079 & Intron & $0.27(\mathrm{C})$ & $\mathrm{PC}$ & 0.90 & 1080 & Jacobs et al., 2008 \\
\hline
\end{tabular}

$\mathrm{MAF}$, minor allele frequency; ECM, extracellular matrix; *OR, corresponding to the minor homozygous genotype in a codominant model; $n$-size, cases + controls; OA, osteoarthritis; AR, rheumatoid arthritis; PC, prostate cancer; BC, breast cancer; ACL, anterior cruciate ligament. All studies were case-control design. 
using the AccuFill system (Thermo Fisher Scientific). Amplification was conducted following the manufacturer's protocol. Raw results were analyzed with the QuantStudio 12K Flex software v1.2.2.

\section{Statistical analysis}

The clinical variables were evaluated with Student's $t$-test or Fisher's exact test, when appropriate, and values were expressed as mean $\pm \mathrm{SD}$. Gene and allele frequencies of all polymorphisms were calculated and compared between cases and controls using Fisher's exacts test. In order to control the global false positive rate, only SNPs with a statistically significant $p$-value in Fisher's exact test were considered in the multivariate analysis. Associations of each SNP with OA risk were assessed with logistic regression models adjusted by age, gender, BMI, and ancestry, taking into account a codominant inheritance model for the SNP. The Bonferroni's test was used to determine the significance level to correct multiple test errors, which taking into account the nine selected SNPs, an adjusted $p$-value $<0.005$ $(0.05 / 9)$ was considered statistically significant. HardyWeinberg equilibrium (HWE) in controls was calculated using the chi-squared test.

We used the STRUCTURE software v2.3.4 (Pritchard Lab, Stanford University, USA) to evaluate the effect of population stratification on the associations found of each population $\mathrm{k}(\mathrm{k}=3)$ with the genotypes of the nine AIMs mentioned above (Table S1). This information was included in the logistic regression models to adjust the associations found between the studied polymorphism and OA by individual mix.

The effect of the associated polymorphisms on groups stratified by gender and age (early OA, $<50$ years; late OA, $>50$ years) was analyzed (Table S2). All statistical analyses were performed with the STATA v14.0 (StataCorp, Texas, USA) statistical package considering an $\alpha=0.05$ significance level.

In order to study the epistasis, we used the MDR v3.0.2 or GMDR v0.9 statistical packages with Ritchie's algorithm (Ritchie et al., 2003). To corroborate the interaction model proposed by the MDR method, a logistic regression model was carried out, which included the SNPs suggested by the MDR. Subsequently, the $p$-value of the model, the adjusted $\mathrm{r}^{2}$, as well as the $p$-values for the ORs of the SNPs, were evaluated (Table S3).

\section{Results}

\section{Characteristics of the study population}

Table 2 shows the demographic and clinical characteristics of cases $(n=92)$ and controls $(n=147)$. Cases were significantly older than controls $(47.2 \pm 12.4$ vs. $40.9 \pm 12.0$ years, $p=0.0001)$. Gender ratio was similar among the study groups, with women being predominant in both. BMI showed overweight in OA patients, whereas the control group showed normal weight, albeit on the upper threshold. The majority of cases and controls were from Mexico City (77.2\% and $84.3 \%$, respectively).

\section{Genetic and allelic frequencies of the SNPs studied in OA patients and controls}

After adjusting for age, gender, BMI and ancestry, there were no significant differences between the genotype or allele frequencies of the analyzed polymorphisms in the study groups. Genotype distributions were compatible with HWE in controls, except for the HIFIAN (rs11292) polymorphism (Table 3).

\section{Polymorphisms distribution in groups stratified by gender and age groups}

The polymorphism distributions stratified by gender and age are shown in Table S2. In men, the GA, CA, and GA heterozygote genotypes of the COL3A1 (rs1800255), $V E G F A$ (rs699947), and EGF (rs4444903) polymorphisms, respectively, were associated with a decreased OA risk (OR $=0.07,95 \% \mathrm{CI}=0.00-0.84, p=0.03 ; \mathrm{OR}=0.04,95 \% \mathrm{CI}=$ $0.00-0.85, p=0.03 ; \mathrm{OR}=0.10,95 \% \mathrm{CI}=0.01-0.94, p=$ 0.04 , respectively). Regarding the analysis stratified by age groups, the CT heterozygote genotype of the MMP3 (rs679620) polymorphism was associated with an increased risk of developing $\mathrm{OA}$ among the $\leq 50$ year-old group $(\mathrm{OR}=$ $3.03,95 \% \mathrm{CI}=1.07-8.93, p=0.03)$, whereas the $\mathrm{G}$ minor

Table 2 - Characteristics of the study population.

\begin{tabular}{|c|c|c|c|c|}
\hline Parameter & Total $(\mathrm{n}=239)$ & $\mathrm{OA}(\mathrm{n}=92)$ & Controls $(n=147)$ & $p$ \\
\hline Age (years) & $43.4 \pm 12.5$ & $47.2 \pm 12.4$ & $40.9 \pm 12.0$ & 0.0001 \\
\hline \multicolumn{5}{|l|}{ Gender } \\
\hline Female (\%) & $185(77.4)$ & $80(87.0)$ & $105(71.4)$ & \multirow[t]{2}{*}{$0.005 *$} \\
\hline Male $(\%)$ & $54(22.6)$ & $12(13.0)$ & $42(28.6)$ & \\
\hline $\mathrm{BMI}\left(\mathrm{Kg} / \mathrm{cm}^{2}\right)$ & $26.5 \pm 4.76$ & $29.0 \pm 4.19$ & $24.8 \pm 4.38$ & $<0.0001$ \\
\hline \multicolumn{5}{|l|}{ Place of birth } \\
\hline Mexico City & $195(81.5)$ & $71(77.2)$ & $124(84.3)$ & \multirow[t]{2}{*}{$0.40^{*}$} \\
\hline Others states of Mexico (central region) & $44(18.5)$ & $21(22.8)$ & $23(15.7)$ & \\
\hline
\end{tabular}

Data are expressed as mean \pm SD. $p$-values were estimated using Students $t$-test, $\alpha=0.05 ; p^{*}$-values were estimated using Fisher's exact test, $\alpha=0.05$. BMI, body-mass index, normal: $18.5-24.9$; overweight: $25.0-29.9$; obesity: $\geq 30.0$. Significant $p$-values are in bold. 
Table 3 - Genetic and allelic frequencies of SNPs studied in OA patients and controls.

\begin{tabular}{|c|c|c|c|c|c|c|}
\hline Gene (SNP rs ID) & OA N $(\%)$ & Controls N $(\%)$ & $\mathrm{OR}^{*}$ & $(95 \% \mathrm{CI})$ & $p$ & HWE in controls \\
\hline \multicolumn{7}{|l|}{$\begin{array}{l}\text { VEGFA } \\
(\mathrm{rs} 3025039)\end{array}$} \\
\hline $\mathrm{CC}$ & $31(38.7)$ & $57(47.9)$ & 1.00 & Reference & & \\
\hline $\mathrm{CT}$ & $37(46.2)$ & $44(37.0)$ & 1.48 & $(0.76-2.88)$ & 0.25 & 0.061 \\
\hline $\mathrm{TT}$ & $12(15.0)$ & $18(15.1)$ & 1.23 & $(0.50-3.03)$ & 0.64 & \\
\hline $\mathrm{T}$ & $61(38.1)$ & $79(33.6)$ & 1.20 & $(0.77-1.88)$ & 0.41 & \\
\hline \multicolumn{7}{|l|}{ VEGFA (rs699947) } \\
\hline $\mathrm{CC}$ & $39(48.1)$ & $57(50.0)$ & 1.00 & Reference & & 0.063 \\
\hline $\mathrm{CA}$ & $34(42.0)$ & $41(36.0)$ & 1.03 & $(0.50-2.12)$ & 0.93 & \\
\hline AA & $8(9.90)$ & $16(14.0)$ & 0.54 & $(0.17-11.7)$ & 0.28 & \\
\hline A & $50(30.9)$ & $73(32.0)$ & 0.81 & $(0.49-1.35)$ & 0.43 & \\
\hline \multicolumn{7}{|l|}{$H I F 1 A N(\operatorname{rs} 11292)$} \\
\hline $\mathrm{AA}$ & $61(81.3)$ & $51(58.6)$ & 1.00 & Reference & & 0.011 \\
\hline AG & $0(0.00)$ & $25(28.7)$ & - & - & - & \\
\hline GG & $14(18.7)$ & $11(12.6)$ & 0.68 & $(0.25-1.78)$ & 0.43 & \\
\hline G & $28(18.7)$ & $47(27.0)$ & 0.58 & $(0.30-1.09)$ & 0.09 & \\
\hline \multicolumn{7}{|l|}{$\begin{array}{l}\text { COL3A1 } \\
(\mathrm{rs} 1800255)\end{array}$} \\
\hline GG & $35(41.7)$ & $30(40.5)$ & 1.00 & Reference & & 0.102 \\
\hline GA & $42(50.0)$ & $39(52.7)$ & 1.36 & $(0.63-2.92)$ & 0.42 & \\
\hline AA & $7(8.33)$ & $5(6.76)$ & 2.20 & $(0.41-11.7)$ & 0.35 & \\
\hline G & $112(66.7)$ & $99(66.9)$ & 1.00 & Reference & & \\
\hline A & $56(33.3)$ & $49(33.1)$ & 1.29 & $(0.76-2.20)$ & 0.33 & \\
\hline \multicolumn{7}{|l|}{$E G F(\mathrm{rs} 4444903)$} \\
\hline GG & $23(28.0)$ & $31(27.9)$ & 1.00 & Reference & & 0.890 \\
\hline GA & $41(50.0)$ & $56(50.5)$ & 1.04 & $(0.48-2.27)$ & 0.90 & \\
\hline AA & $18(22.0)$ & $24(21.6)$ & 1.51 & $(0.58-3.91)$ & 0.39 & \\
\hline A & $77(47.0)$ & $104(46.8)$ & 1.20 & $(0.75-1.92)$ & 0.42 & \\
\hline \multicolumn{7}{|l|}{$M M P 3$ (rs679620) } \\
\hline $\mathrm{CC}$ & $39(48.7)$ & $41(51.9)$ & 1.00 & Reference & & 0.073 \\
\hline $\mathrm{CT}$ & $30(37.5)$ & $27(34.2)$ & 2.17 & $(0.93-5.08)$ & 0.07 & \\
\hline $\mathrm{TT}$ & $11(13.8)$ & $11(13.9)$ & 0.88 & $(0.32-2.41)$ & 0.81 & \\
\hline $\mathrm{T}$ & $52(32.5)$ & $49(31.0)$ & 1.09 & $(0.64-1.87)$ & 0.73 & \\
\hline \multicolumn{7}{|l|}{$\begin{array}{l}M M P 13 \\
\text { (rs2252070) }\end{array}$} \\
\hline $\mathrm{TT}$ & $38(44.7)$ & $46(48.9)$ & 1.00 & Reference & & 0.602 \\
\hline $\mathrm{TC}$ & $41(48.2)$ & $41(43.6)$ & 1.18 & $(0.62-2.26)$ & 0.60 & \\
\hline $\mathrm{CC}$ & $6(7.10)$ & $7(7.50)$ & 1.09 & $(0.32-3.71)$ & 0.89 & \\
\hline $\mathrm{C}$ & $53(31.2)$ & $55(29.3)$ & 1.09 & $(0.68-1.76)$ & 0.70 & \\
\hline \multicolumn{7}{|l|}{ NOS2 (rs2297518) } \\
\hline GG & $65(81.2)$ & $66(80.5)$ & 1.00 & Reference & & 0.887 \\
\hline GA & $14(17.5)$ & $15(18.3)$ & 0.86 & $(0.37-2.03)$ & 0.75 & \\
\hline AA & $1(1.25)$ & $1(1.22)$ & 1.51 & $(0.08-26.0)$ & 0.78 & \\
\hline A & $16(10.0)$ & $17(10.4)$ & 0.94 & $(0.44-2.01)$ & 0.89 & \\
\hline \multicolumn{7}{|l|}{ NOS3 (rs2070744) } \\
\hline $\mathrm{TT}$ & $58(66.7)$ & $53(63.1)$ & 1.00 & Reference & & 0.092 \\
\hline $\mathrm{TC}$ & $23(26.4)$ & $24(28.6)$ & 0.89 & $(0.42-1.86)$ & 0.77 & \\
\hline $\mathrm{CC}$ & $6(6.90)$ & $7(8.33)$ & 0.64 & $(0.19-2.17)$ & 0.48 & \\
\hline $\mathrm{C}$ & $35(20.1)$ & $38(22.6)$ & 0.80 & $(0.46-1.40)$ & 0.45 & \\
\hline
\end{tabular}

*The multi-variable model was adjusted for age (continuous data), gender (male, female), BMI (continous data) and admixture; OA, osteoarthritis patients; OR, Odds ratio; CI, confidence interval. HWE, Hardy-Weinberg equilibrium; if $p<0.05$, it is not consistent with HWE. Significant $p$-values are in bold. 
allele of the HIFIAN (rs11292) polymorphism was associated with protection in the same age group $(\mathrm{OR}=0.44,95 \%$ $\mathrm{CI}=0.22-0.88, p=0.02)$. The AA homozygote genotype of the VEGFA (rs699947) polymorphism showed an association with protection for the over 50 year-old age group $(\mathrm{OR}=$ $0.06,95 \% \mathrm{CI}=0.00-0.86, p=0.03)$.

\section{MDR analysis}

Table 4 summarizes the results of the MDR analysis, which assesses all possible combinations of the associated polymorphisms stratified by gender and age. According to the MDR analysis, the best model included the $M M P 3$ (rs679620), COL3A1 (rs1800255), and HIF1AN (rs11292) polymorphisms. This model had a balanced accuracy test of 0.6434 , a consistency of cross-validation of $10 / 10$, and an interaction $p$-value $=0.0010$. Additionally, $p$-values from logistic regression models were included.

Figure 1 shows the interaction map of the studied polymorphisms, based on entropy measures among individual variables. A strong interaction effect was observed between MMP3 (rs679620) and COL3A1 (rs1800255) polymorphisms, and between COL3A1 (rs1800255) and VEGFA (rs699947) polymorphisms, with information gain values of $3.21 \%$ and $2.34 \%$, respectively. Significance value was also confirmed through logistic regression models, with an interaction $p$-value $=0.036$. Additionally, in our model we found interactions in high-risk genotypes of the HIFIAN, MMP3 and COL $3 A 1$ genes, the most representative were $[\mathrm{AA}+\mathrm{CC}+\mathrm{GA}],[\mathrm{AA}+\mathrm{CT}+\mathrm{GA}]$ and $[\mathrm{AA}+\mathrm{CT}+\mathrm{GG}]$, respectively; and low-risk genotypes [AA+CC+GG], $[\mathrm{GG}+\mathrm{TT}+\mathrm{GA}]$ and $[\mathrm{AA}+\mathrm{TT}+\mathrm{GA}]$, respectively (Figure 2 ).

\section{Discussion}

In this study, we applied the MDR computation method to assess the epistasis of candidate genes related to articular cartilage ECM in knee OA patients in a Mexican population. Our main findings revealed important gene-gene interactions between the MMP3 (rs679620), VEGFA (rs699947), and COL3A1 (rs1800255) polymorphisms.

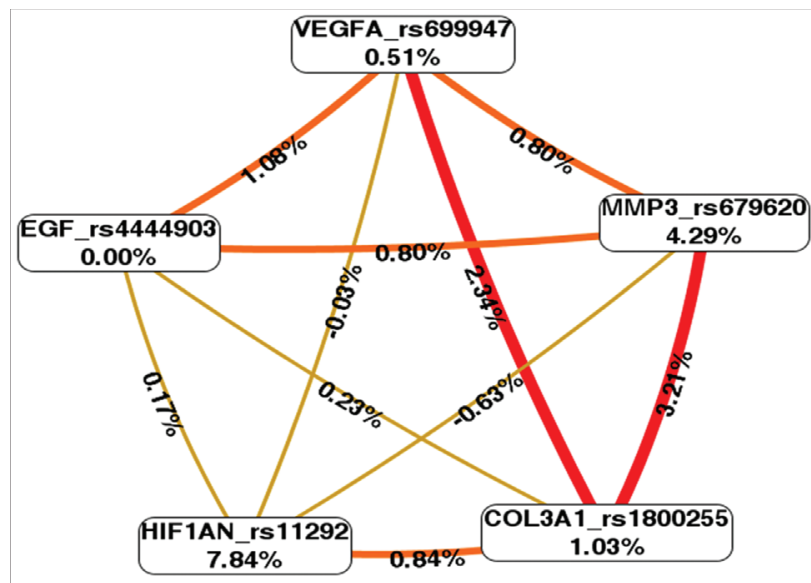

Figure 1 - Interaction map for osteoarthritis risk. The interaction model describes the percentage of the entropy (information gain) that is explained by each factor or 2-way interaction. Values inside nodes indicate information gain of individual attributes or main effects, whereas values between nodes show information gain of pairwise combinations of attributes or interaction effects. Positive entropy (plotted in red or orange) indicates interaction, which can be interpreted as a synergistic or nonadditive relationship; while negative entropy (plotted in green-yellow) indicates independence or additivity (redundancy).

The importance of analyzing MMP-3 (stromelysin-1) is that it is a zinc-dependent endopeptidase that catalytically degrades several collagen and non-collagen proteins of the basement membrane and the ECM. It is considered an essential regulator of ECM homeostasis in healthy and damaged musculoskeletal soft tissue (Gibbon et al., 2017). The MMP3 gene has several genetic polymorphisms that could considerably affect ECM degradation and remodeling. Cases and controls studies evaluating different SNPs within the MMP3 gene have been conducted, including the rs679620 variant, yet none has been associated with OA risk (Guo et al., 2017; Tong et al., 2017). The non-association of this polymorphism that we observed in our work is consistent with previous reports, and it was unexpected, considering its functional role and the active involvement of this metalloprotease in ECM degradation in OA. This phenome-

Table 4 - MDR analysis

\begin{tabular}{|c|c|c|c|c|c|c|}
\hline Locus number & Model & Training Bal Acc & Testing Bal Acc & Cross-validation consistency & $p$ & $p^{*}$ \\
\hline 1 & HIF1AN_rs11292 & 0.5963 & 0.5391 & $6 / 10$ & 0.3770 & 0.028 \\
\hline 2 & MMP3_rs679620, HIF1AN_rs11292 & 0.6390 & 0.6053 & $9 / 10$ & 0.0547 & 0.085 \\
\hline 3 & $\begin{array}{l}\text { MMP3_rs679620, COL3A1_rs1800255, } \\
\text { HIF1AN_rs11292 }\end{array}$ & 0.6847 & 0.6434 & $10 / 10$ & 0.0010 & 0.196 \\
\hline 4 & $\begin{array}{l}\text { MMP3_rs679620, COL3A1_rs1800255, } \\
\text { VEGFA_699947, HIF1AN_rs11292 }\end{array}$ & 0.7107 & 0.5614 & $7 / 10$ & 0.1719 & 0.408 \\
\hline 5 & $\begin{array}{l}\text { MMP3_rs679620, COL3A1_rs1800255, } \\
\text { VEGFA_699947, HIF1AN_rs11292, } \\
\text { EGF rs4444903 }\end{array}$ & 0.7422 & 0.4674 & $10 / 10$ & 0.8281 & 0.356 \\
\hline
\end{tabular}

$p$-values were based on 1000 permutations and were obtained from sign test by MDR method.

$* p$-values were obtained from logistic regression models.

MDR, multifactor dimensionality reduction; Testing Bal Acc, testing-balanced accuracy.

Significant $p$-values are in bold. 


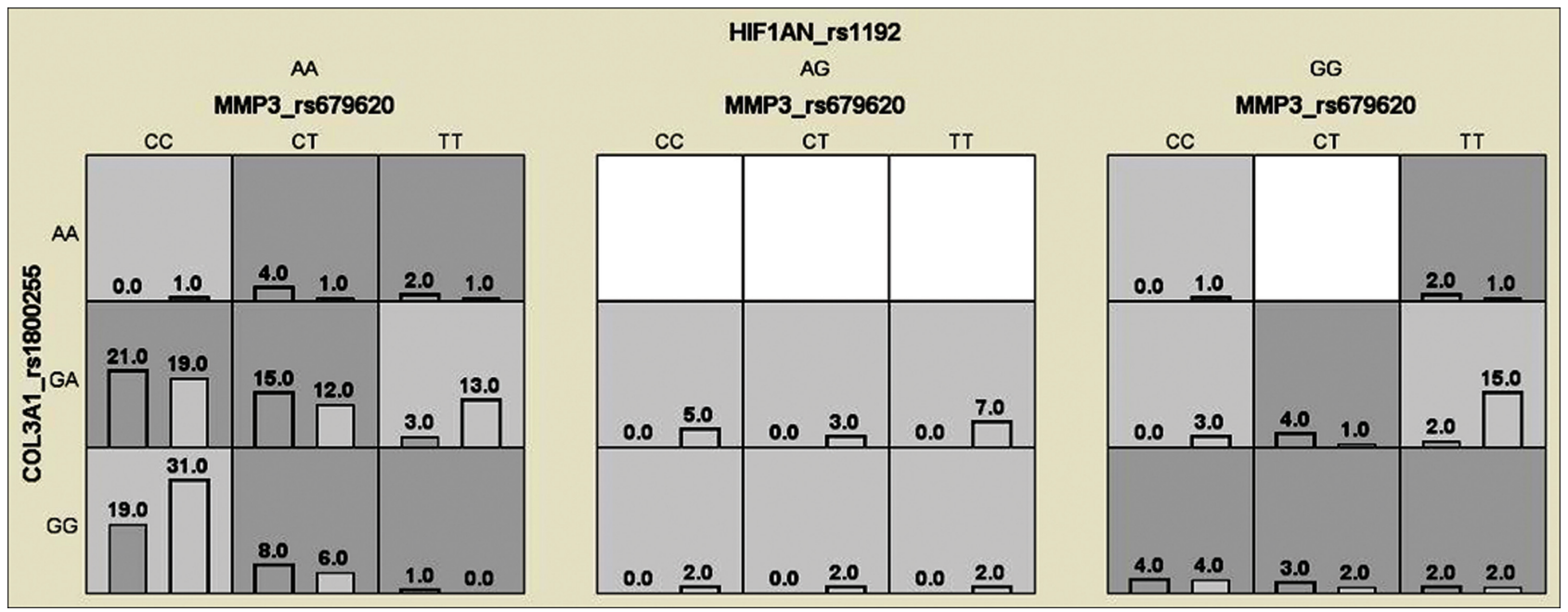

Figure 2 - Distribution of high-risk and low-risk genotypes in the best three-locus model. The distribution shows high-risk (dark shading) and low-risk (light shading) genotypes associated with knee OA in the three-locus interaction detected by MDR analysis. The percentage of osteoarthritic subjects (left black bar in boxes) and control subjects (right hatched bar in boxes) is shown for each three-locus genotype combination. Boxes were labeled as high-risk if the ratio of the percentage of cases to controls met or exceeded the threshold of 1.0. Boxes were labeled as low-risk if the threshold was not exceeded. Based on the pattern of high-risk and low-risk genotypes, this three-locus model is evidence of gene to gene interaction.

non has been reported in other pathologies, such as rheumatoid arthritis (Rodriguez-Lopez et al., 2006). However, when we stratified by age group, we observed that carriers of the CT heterozygote genotype showed a significant association with OA risk in people younger than 50 years old, which might suggest the possibility that the polymorphism could be associated with a more aggressive disease phenotype or more rapid progression.

On the other hand, it has been proved that an increase in the VEGF protein level is associated with OA progression. Apparently, VEGF is involved in specific OA processes, including cartilage degeneration, osteophyte formation, subchondral bone cysts, sclerosis, synovitis, and pain. Moreover, inhibition of VEGF signaling decreases OA progression (Mariani et al., 2014; Hamilton et al., 2016). Genetically, there is little evidence of the association of $V E G F A$ polymorphisms in OA pathogenesis. Sánchez-Enríquez et al. (2008) assessed the $-460 \mathrm{~T} / \mathrm{C}(\mathrm{rs} 833061)$ and $+405 \mathrm{C} / \mathrm{G}$ (rs2010963) polymorphisms of VEGFA in knee arthrosis patients and compared them to healthy controls, but they did not find a significant association. In our study, we evaluated the rs699947 variant, and even though distribution was similar in cases and controls, we did find a genotype-phenotype association when stratifying by gender and age groups. We observed that the CA heterozygote genotype provides protection against OA development, but only among men, and that the AA homozygote genotype provides protection exclusively to individuals 50 years old or older. This variant is located in a promoter region of the VEGFA gene, but since its functional role in OA pathogenesis is not yet known, further research is needed.

Substitution of type-II collagen with type-III collagen in the articular cartilage ECM is a common feature of OA. Studies based on mRNA analysis prove that type-III collagen expression is associated with type-II collagen expres- sion, which suggests a metabolic reaction of the chondrocytes to type-III collagen build-up in articular cartilage areas, probably as a response to mechanical injury or other damage to the ECM (Hosseininia et al., 2016). To date, there is no scientific evidence of an association between polymorphisms of the COL3A1 gene with knee OA. StepienSlodkowska et al. (2015) evaluated the rs1800255 variant among Polish patients with an anterior cruciate ligament injury and observed that the distribution of the AA homozygote genotype was higher in comparison to the control group $(p=0.0087)$. In our study, we evaluated the same variant among knee OA patients, and we found that the GA heterozygote genotype is significantly associated with protection, but just among men. This variant causes a change in Ala531Thr, but its role at the articular cartilage level is unknown. There might be a defect in procollagen III synthesis, resulting in a structural modification of the protein.

By using MDR analysis, we found evidence of two strong interactions with significant value between three out of nine variants of the eight candidate genes involved in ECM degradation that we assessed. Likewise, we could identify interactions between the $\operatorname{rs} 11292$ (HIFIAN), rs679620 (MMP3) and rs1800255 (COL3A1) polymorphisms, generating high and low risk haplotypes between OA patients. On the other hand, when we performed the interaction analysis using logistic regression models, we did not detect any interaction. One of the virtues of the MDR method is that it does not require very large sample sizes to detect interactions. However, logistic regression models require a very large " $\mathrm{N}$ " to detect statistical significance. Also, polymorphisms that are poorly represented or of low frequency are difficult to detect, and interactions between the polymorphisms can only be evaluated by pairs.

It is well known that OA pathogenesis is multifactorial, and its complexity is primarily due to its polygenic na- 
ture. Given this polygenic nature, it has been difficult to prove gene-gene interactions associated with knee OA. To date, only four studies have reported gene-gene interactions in knee OA susceptibility. The first of these studies evaluated the TGF- $\beta /$ Smad 3 signaling pathway using the MDR method (Su et al., 2015). Other two focused on the Wnt/ $\beta$ catenin signaling pathway and on the uric acid transporters polymorphisms, using logistic regression models (Fernández-Torres et al., 2018a,b). The most recent one published by our working group, identified interactions between polymorphisms of the $A D I P O Q$ and $P O N 1$ genes, using the MDR method (Fernández-Torres et al., 2019). The effect magnitude of any polymorphism is likely to be overlooked if the genes are individually examined, without considering potential interactions with other genes, especially those in related pathways.

Despite the results and the advantages offered by the MDR method, it is important to emphasize some aspects. Unlike classical genetic analysis, our main approach highlights the importance of evaluating in an integral manner the effect of genetic variants in knee OA. However, we are aware of certain limitations of our study, the first being that our sample size is limited. However, we believe that after performing a multivariate analysis and a rigorous selection of our patients and controls, the presented data reinforce the biological plausibility of the SNPs in the OA. Second, our association study was limited to one population, so more studies in different populations are needed to support our findings, as well as to evaluate the functionality of the associated SNPs and be able to show evidence of whether they have a causal effect or not. Finally, there are more variants of the same gene that were not analyzed, as well as other genes of the ECM that were not considered and whose impact on OA development is unknown. Therefore, these preliminary results must be interpreted with caution, regarding our observation of a non-association between some polymorphisms and knee OA.

In summary, we evaluated nine polymorphisms associated with components of the articular cartilage ECM in knee OA patients in a Mexican population using the MDR and GMDR methods. The rs679620 (MMP3), rs699947 (VEGFA), and rs 1800255 (COL3A1) polymorphisms showed epistasis. Knowing the interactions involved in ECM metabolism could provide a new tool to identify individuals at high risk of developing knee OA. Further studies are needed to establish the mechanisms of interaction between these polymorphisms and their effects on knee OA susceptibility. This work highlights the importance to open the possibility of evaluating other pathways involved in ECM metabolism, such as the bone morphogenetic protein (BMP), fibroblast growth factor (FGF), hypoxia-inducible factor (HIF), nuclear factor kappa-B (NF-кB), mitogenactivated protein kinase (MAPK), and hedgehog (Hh) signaling pathways.

\section{Acknowledgments}

This work was supported by federal resources of the INR-LGII. The authors thank Alberto López-Reyes for supporting the purchase of the genotyping chip.

\section{Conflict of Interests}

The authors declared no potential conflicts of interest with respect to the research, authorship, and/or publication of this article.

\section{Author Contributions}

JFT conceived the study, sample processing, interpretation of results, writing first draft of manuscript and final manuscript approval. GAMN performed the statistical analysis, and reviewed the manuscript draft. YZC recruited healthy subjects and applied the questionnaire. CL recruited and clinically evaluated osteoarthritis patients. DGR performed the open array processing and allelic discrimination analysis. KMF was involved in study design and interpretation of results. All authors reviewed and approved the final version of the manuscript.

\section{References}

Abramson SB (2008a) Osteoarthritis and nitric oxide. Osteoarthritis Cartilage 16:S15-S20.

Abramson SB (2008b) Nitric oxide in inflammation and pain associated with osteoarthritis. Arthritis Res Ther 10:S2.

Altman R, Asch E, Bloch D, Bole G, Borenstein D, Brandt K, Christy W, Cooke TD, Greenwald R, Hochberg M et al. (1986) Development of criteria for the classification and reporting of osteoarthritis. Classification of osteoarthritis of the knee. Diagnostic and therapeutic criteria committee of the American Rheumatism Association. Arthritis Rheum 29:1039-1049.

Arden N and Nevitt M (2006) Osteoarthritis: Epidemiology. Best Pract Res Clin Rheumatol 20:3-25.

Bertrand J, Cromme C, Umlauf D, Frank S and Pap T (2010) Molecular mechanisms of cartilage remodelling in osteoarthritis. Int J Biochem Cell Biol 42:1594-601.

Bonilla C, Parra EJ, Pfaff CL, Dios S, Marshall JA, Hamman RF, Ferrell RE, Hoggart CL, McKeigue PM and Shriver MD (2004) Admixture in the Hispanics of the San Luis Valley, Colorado, and its implications for complex trait gene mapping. Ann Hum Genet 68:139-152.

Chen GB, Xu Y, Xu HM, Li MD, Zhu J and Lou XY (2011) Practical and theoretical considerations in study design for detecting gene-gene interactions using MDR and GMDR approaches. PLoS One 6:e16981.

Chen Y and Mattey DL (2012) Age at onset of rheumatoid arthritis: association with polymorphisms in the vascular endothelial growth factor A (VEGFA) gene and an intergenic locus between matrix metalloproteinase (MMP) 1 and 3 genes. Clin Exp Rheumatol 30:894-898.

Cho YM, Ritchie MD, Moore JH, Park JY, Lee KU, Shin HD, Lee HK and Park KS (2004) Multifactor-dimensionality reduction shows a two-locus interaction associated with Type 2 diabetes mellitus. Diabetologia 47:549-554.

Choundry S, Coyle NE, Tang H, Salari K, Lind D, Clark SL, Tsai HJ, Naqvi M, Phong A, Ung N et al. (2006) Population strati- 
fication confounds genetic association studies among Latinos. Hum Genet 118:652-664.

De Filippis L, Gulli S, Caliri A, Romano C, Munaò F, Trimarchi G, La Torre D, Fichera C, Pappalardo A, Triolo G et al. (2004) Epidemiology and risk factors in osteoarthritis: literature review data from "OASIS" study. Reumatismo 56:169-84.

Du Y, Lin Y, Yin K, Zhou L, Jiang Y, Yin W and Lu J (2019) Single nucleotide polymorphisms of let-7-related genes increase susceptibility to breast cancer. Am J Transl Res 11:1748-1759.

Fernández-Moreno M, Rego I, Carreira-García V and Blanco FJ (2008) Genetics in Osteoarthritis. Curr Genomics 9:542-547.

Fernández-Torres J, Martínez-Nava GA, Gutiérrez-Ruíz MC, Gómez-Quiroz LE and Gutiérrez M (2017) Role of HIF-1 $\alpha$ signaling pathway in Osteoarthritis: A systematic review. Rev Bras Reumatol Eng Ed 57:162-173.

Fernández-Torres J, Martínez-Nava GA, Oliviero F, López-Reyes AG, Martínez-Flores K, Garrido-Rodríguez D, FranciscoBalderas A and Zamudio-Cuevas Y (2018a) Common gene variants interactions related to uric acid transport are associated with knee osteoarthritis susceptibility. Connect Tissue Res 60:219-229

Fernández-Torres J, Zamudio-Cuevas Y, López-Reyes A, Garrido-Rodríguez D, Martínez-Flores K, Lozada CA, MuñózValle JF, Oregon-Romero E and Martínez-Nava GA (2018b) Gene-gene interactions of the Wnt $/ \beta$-catenin signaling pathway in knee osteoarthritis. Mol Biol Rep 45:1089-1098.

Fernández-Torres J, Martínez-Nava GA, Zamudio-Cuevas Y, Martínez-Flores K and Espinosa-Morales R (2019) Epistasis between ADIPOQ rs1501299 and PON1 rs662 polymorphisms is potentially associated with the development of knee osteoarthritis. Mol Biol Rep 46:2049-2058.

Gibbon A, Hobbs H, van der Merwe W, Raleigh SM, Cook J, Handley CJ, Posthumus M, Collins M and September AV (2017) The MMP3 gene in musculoskeletal soft tissue injury risk profiling: A study in two independent sample groups. J Sports Sci 35:655-662.

Gui J, Andrew AS, Andrews P, Nelson HM, Kelsey KT, Karagas MR and Moore JH (2011) A robust multifactor dimensionality reduction method for detecting gene-gene interactions with application to the genetic analysis of bladder cancer susceptibility. Ann Hum Genet 75:20-28.

Guo W, Xu P, Jin T, Wang J, Fan D, Hao Z, Ji Y, Jing S, Han C, Du $\mathrm{J}$ et al. (2017) MMP-3 gene polymorphisms are associated with increased risk of osteoarthritis in Chinese men. Oncotarget 8:79491-79497.

Hahn LW, Ritchie MD and Moore JH (2003) Multifactor dimensionality reduction software for detecting gene-gene and geneenvironment interactions. Bioinformatics 19:376-382.

Hamilton JL, Nagao M, Levine BR, Chen D, Olsen BR and Im HJ (2016) Targeting VEGF and Its Receptors for the Treatment of Osteoarthritis and Associated Pain. J Bone Miner Res 31:911-924.

Hernández-Cáceres AE, Rodriguez-Amado J, Peláez-Ballestas I, Vega-Morales D and Garza-Elizondo MA (2015) Factors associated with treatment of osteoarthritis: Analysis of a COPCORD study in Nuevo León, México. Reumatol Clin 11:204-209.

Hosseininia S, Weis MA, Rai J, Kim L, Funk S, Dahlberg LE and Eyre DR (2016) Evidence for enhanced collagen type III deposition focally in the territorial matrix of osteoarthritic hip articular cartilage. Osteoarthritis Cartilage 24:1029-1035.

Jacobs EJ, Hsing AW, Bain EB, Stevens VL, Wang Y, Chen J, Chanock SJ, Zheng SL, Xu J, Thun MJ et al. (2008) Poly- morphisms in angiogenesis-related genes and prostate cancer. Cancer Epidemiol Biomarkers Prev 17:972-977.

Kaynak M, Nijman F, van Meurs J, Reijman M and Meuffels DE (2017) Genetic variants and anterior cruciate ligament rupture: A systematic review. Sports Med 47:1637-1650.

Kellgren JH and Lawrence JS (1957) Radiological assessment of osteo-arthritis. Ann Rheum Dis 16:494-502.

Kohli S, Kumar R, Gupta M, Tyagi S and Pasha MA (2016) Impact of interactions between risk alleles on clinical endpoints in hypertension. Heart Asia 8:83-89.

Kraus VB, Blanco FJ, Englund M, Karsdal MA and Lohmander LS (2015) Call for standardized definitions of osteoarthritis and risk stratification for clinical trials and clinical use. Osteoarthritis Cartilage 23:1233-1241.

Long DL, Ulici V, Chubinskaya S and Loeser RF (2015) Heparin-binding epidermal growth factor-like growth factor (HB-EGF) is increased in osteoarthritis and regulates chondrocyte catabolic and anabolic activities. Osteoarthritis Cartilage 23:1523-1531.

Lou XY, Chen GB, Yan L, Ma JZ, Zhu J, Elston RC and Li MD (2007) A generalized combinatorial approach for detecting gene-by-gene and gene-by-environment interactions with application to nicotine dependence. Am J Hum Genet 80:1125-1137.

Mariani E, Pulsatelli L and Facchini A (2014) Signaling pathways in cartilage repair. Int J Mol Sci 15:8667-8698.

Martel-Pelletier J, Boileau C, Pelletier JP and Roughley PJ (2008) Cartilage in normal and osteoarthritis conditions. Best Pract Res Clin Rheumatol 22:351-384

Mechanic LE, Luke BT, Goodman JE, Chanock SJ and Harris CC (2008) Polymorphism Interaction Analysis (PIA): A method for investigating complex gene-gene interactions. BMC Bioinformatics 9:146.

Motsinger AA and Ritchie MD (2006) Multifactor dimensionality reduction: an analysis strategy for modelling and detecting gene-gene interactions in human genetics and pharmacogenomics studies. Hum Genomics 2:318-28.

Moore JH and Andrews PC (2015) Epistasis analysis using multifactor dimensionality reduction. Methods Mol Biol 1253:301-314.

Oswald ES, Chao PH, Bulinski JC, Ateshian GA and Hung CT (2008) Dependence of zonal chondrocyte water transport properties on osmotic environment. Cell Mol Bioeng 1:339348.

Ozcan SS, Korkmaz M, Balbaloglu O, Percin F, Yilmaz N, Erdogan Y and Gunaydin I (2017) Polymorphisms in the Growth Differentiation Factor 5 (GDF 5) Gene in knee osteoarthritis. J Coll Physicians Surg Pak 27:602-605.

Peláez-Ballestas I, Sanin LH, Moreno-Montoya J, Alvarez-Nemegyei J, Burgos-Vargas R, Garza-Elizondo M, RodríguezAmado J, Goycochea-Robles MV, Madariaga M, Zamudio J et al. (2011) Epidemiology of the rheumatic diseases in Mexico. A study of 5 regions based on the COPCORD methodology. J Rheumatol Suppl 86:3-8.

Pfander D, Swoboda B and Cramer T (2006) The role of HIF1 alpha in maintaining cartilage homeostasis and during the pathogenesis of osteoarthritis. Arthritis Res Ther 8:104.

Ritchie MD, Hahn LW and Moore JH (2003) Power of multifactor dimensionality reduction for detecting gene-gene interactions in the presence of genotyping error, missing data, phenocopy, and genetic heterogeneity. Genet Epidemiol 24:150-157.

Rodriguez-Lopez J, Perez-Pampin E, Gomez-Reino JJ and Gonzalez A (2006) Regulatory polymorphisms in extracellular matrix protease genes and susceptibility to rheumatoid arthritis: a case-control study. Arthritis Res Ther 8:R1. 
Sánchez-Enríquez S, Torres-Carrillo NM, Vázquez-Del Mercado M, Salgado-Goytia L, Rangel-Villalobos H and Muñoz-Valle JF (2008) Increase levels of apo-A1 and apo B are associated in knee osteoarthritis: lack of association with VEGF-460 T/C and $+405 \mathrm{C} / \mathrm{G}$ polymorphisms. Rheumatol Int 29:63-68.

Sophia Fox AJ, Bedi A and Rodeo SA (2009) The basic science of articular cartilage: structure, composition, and function. Sports Health 1:461-468.

Stepien-Slodkowska M, Ficek K, Maciejewska-Karlowska A, Sawczuk M, Zietek P, Król P, Zmijewski P, Pokrywka A and Cieszczyk P (2015) Overrepresentation of the COL3A1 AA genotype in Polish skiers with anterior cruciate ligament injury. Biol Sport 32:143-147.

Ströbel S, Loparic M, Wendt D, Schenk AD, Candrian C, Lindberg RL, Moldovan F, Barbero A and Martin I (2010) Anabolic and catabolic responses of human articular chondrocytes to varying oxygen percentages. Arthritis Res Ther 12:R34.

Su SL, Yang HY, Lee HS, Huang GS, Lee CH, Liu WS, Wang CC, Peng YJ, Lai CH, Chen CY et al. (2015) Gene-gene interactions between TGF- $\beta / \mathrm{Smad} 3$ signalling pathway polymorphisms affect susceptibility to knee osteoarthritis. BMJ Open 5:e07931.

Tong Z, Liu Y, Chen B, Yan L and Hao D (2017) Association between MMP3 and TIMP3 polymorphisms and risk of osteoarthritis. Oncotarget 8:83563-83569. van Meurs JB and Uitterlinden AG (2012) Osteoarthritis year 2012 in review: Genetics and genomics. Osteoarthritis Cartilage 20:1470-1476.

Wang T, Liang Y, Li H, Li Ha, He Q, Xe Y, Shen C, Zhang C, Xiang J, Ding J et al. (2016) Single nucleotide polymorphisms and osteoarthritis: An overview and a meta-analysis. Medicine (Baltimore) 95:e2811.

Zhan Z, Chen Y, Wu J, Li H, He Q, Xue Y, Shen C, Zhang C, Xiang J, Ding J et al. (2013) Functional epidermal growth factor gene polymorphisms and risk of gastric cancer. Oncol Lett 5:631-636.

\section{Supplementary material}

The following online material is available for this article:

'Tahle S1 - Ancestry informative markers (AIMs) studied.

Table S2 - Polymorphisms distribution in groups stratified by gender and age groups.

Table S3 - Polymorphism interactions by logistic regression.

Associate Editor: Jorge Lopez-Camelo

License information: This is an open-access article distributed under the terms of the Creative Commons Attribution License (type CC-BY), which permits unrestricted use, distribution and reproduction in any medium, provided the original article is properly cited. 